\title{
The dynamics of drug treatment in epilepsy: an observational study in an unselected population based cohort with newly diagnosed epilepsy followed up prospectively over 11-14 years
}

\author{
S D Lhatoo, J W A S Sander, S D Shorvon
}

\begin{abstract}
Objectives-To study prospectively long term dynamics and patterns of treatment in a population based cohort of patients with newly diagnosed epilepsy.

Methods-564 patients with definite epilepsy entered the UK National General Practice Study of Epilepsy (NGPSE), between 1984 and 1987, and were prospectively followed up for between 11-14 years. Results-Treatment was started in 433

United Kingdom, about 6000 have inadequate seizure control in the long term. About a third of the patients in this group have one or more seizures every month. Only two thirds of these patients with frequent seizures are likely to switch medication to try and achieve better seizure control. There is probably still considerable room for improvement in prescribing practice in the United Kingdom.
\end{abstract} (77\%) patients. Only $15 \%$ of single seizure patients had medication prescribed initially, although due to high seizure recurrence, more than $70 \%$ ultimately received antiepileptic medication. 209/564 patients $(37 \%)$ were on drug therapy for epilepsy at the time of last follow up. $168 / 564$ patients $(30 \%)$ have stayed continuously on medication and another $41 / 564$ patients (7\%) restarted drug therapy because of seizure recurrence, having withdrawn medication. 98/209 (47\%) of those on treatment are known to be in 5 year terminal remission. Phenytoin (29\%) and carbamazepine $(27 \%)$ were the most commonly preferred first line drugs followed by valproate $(15 \%)$. Less than half of treated patients with partial seizures received carbamazepine as a first line drug and less than a third with generalised seizures were prescribed valproate as first choice drug. Nine out of $31(29 \%)$ patients with one or more seizures a week at last follow up had never tried a second drug and only seven $(23 \%)$ had tried four or more drugs. $11 \%$ of all treatment changes involved a new antiepileptic drug. Treatment changes were associated with low terminal remission rates.

Conclusions-Out of 30000 patients with

University College

London, London

WC1N 3BG, UK

$S$ D Lhatoo

J W A S Sander

S D Shorvon

Correspondence to:

Professor J W Sander,

Department of Clinical and

Experimental Epilepsy,

Institute of Neurology,

University College, London,

Queen Square, London

WC1N 3BG, UK

sander@ion.ac.uk

Received 17 January 2001

and in revised form

14 May 2001

Accepted 26 June 2001
(F Neurol Neurosurg Psychiatry 2001;71:632-637)

Keywords: treatment; epilepsy; population based cohort

The most widely followed model of epilepsy management is one of referral from primary care to hospital clinics followed by investigation, treatment, and follow up at primary, secondary, or shared care levels. A description of the evolution of therapy in this model over time data for knowing where to target treatment improvements and for the planning of health care. In a hospital setting, the various facets of this model are easily studied, but the bias towards more complicated cases is likely to present a distorted picture. ${ }^{1}$ Unselected, population based studies provide a more accurate picture of the generality of therapy, in a representative patient sample, although the potential logistical difficulties involved have resulted in a considerable dearth of such studies. ${ }^{1}$ In particular, there have been no previous prospective, long term studies of treatment dynamics in a population based cohort with epilepsy, although treatment characteristics in a community based prevalent population have been reported on before. ${ }^{2}$

The United Kingdom National General Practice Study of Epilepsy (NGPSE), ${ }^{3}$ is a long term population based cohort of newly diagnosed patients followed up prospectively for 11-14 years. It provides an unselected population in which to study treatment patterns.

We report here an observational study of treatment patterns in this cohort, in which we address the following questions: How many patients with newly diagnosed epileptic seizures receive treatment, and who initiated therapy? How many patients change treatment, and when, in the course of the epilepsy? What are the reasons for drug change and which drugs and drug combinations are used initially and how often is treatment switched or added? Is there uniformity in the choice of antiepileptic drugs for different seizure types? at a population level should provide important 


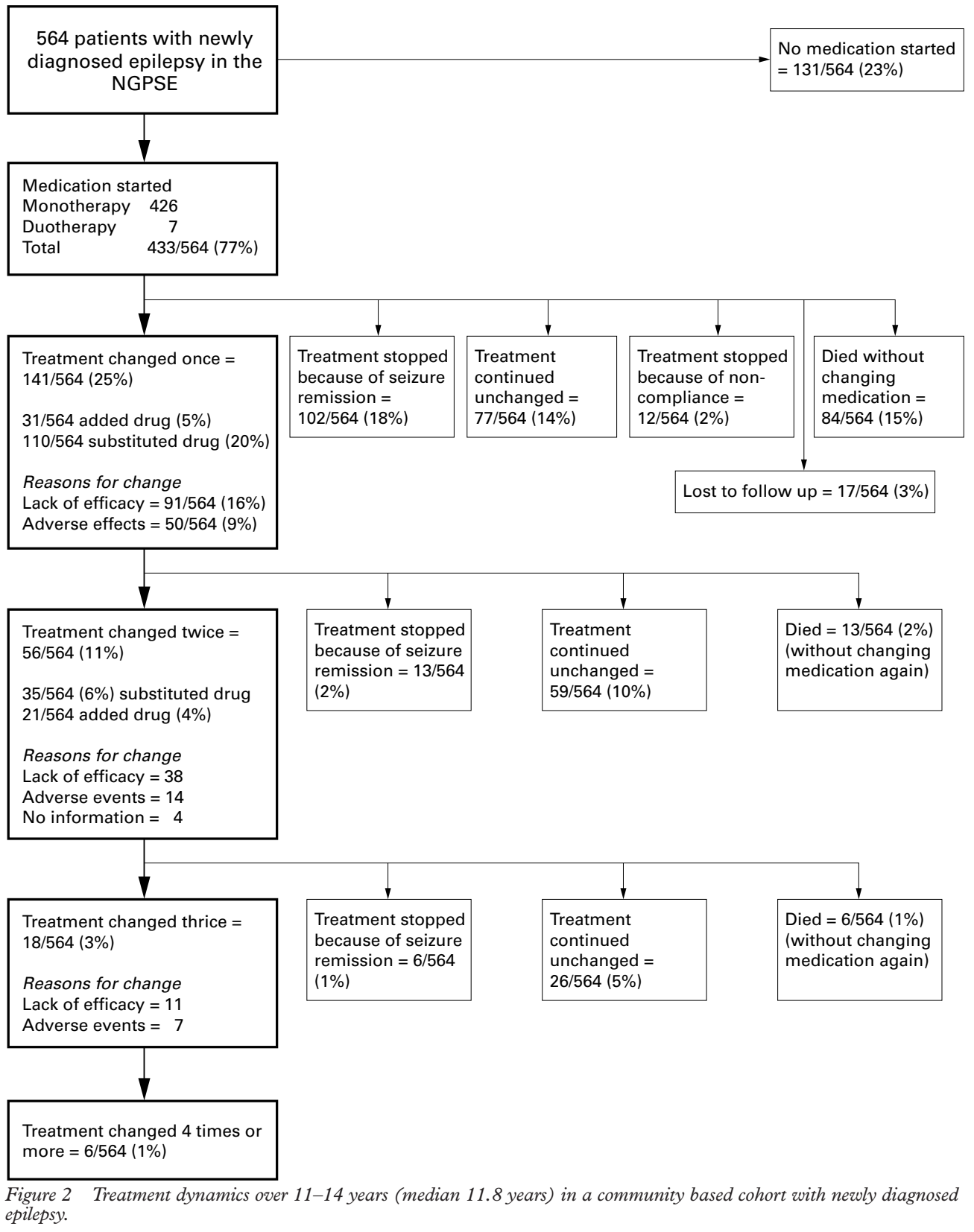

How often were the newer antiepileptic drugs used by an unselected population with epilepsy? To what extent were seizures controlled by drug changes in patients with uncontrolled epilepsy.

\section{Methods}

The methodology of the NGPSE has been described in detail elsewhere. ${ }^{3}$ In brief summary, patients with newly diagnosed epilepsy were identified from 275 general practices throughout the United Kingdom during the years 1984-7. A diagnosis of epilepsy was confirmed by an expert review panel in 564 patients on the basis of information submitted by general practitioners (GPs) and hospital specialists. Epilepsy was defined by the occurrence of one or more definite epileptic seizures regardless of cause. Neonatal seizures, febrile seizures, and attacks in which the diagnosis of

epilepsy was not certain were excluded. All cases were prospectively followed up from the index seizure (the seizure that led to enrolment in the study) by means of regular forms filled in by the GP, and hospital details. The Office for National Statistics flagged all patients, allowing patients to be tracked should they move within the United Kingdom, and notified deaths.

Treatment patterns were analysed in these 564 patients. Data recorded included age, sex, date of index seizure, date of onset of seizures if different from index seizure, seizure type (the Commission on Classification and Terminology of the International League Against Epilepsy, $1981^{4}$ ), person prescribing initial treatment, duration of treatment, number of treatment changes, reasons and types of changes, use of newer antiepileptic drugs, and seizure status. 


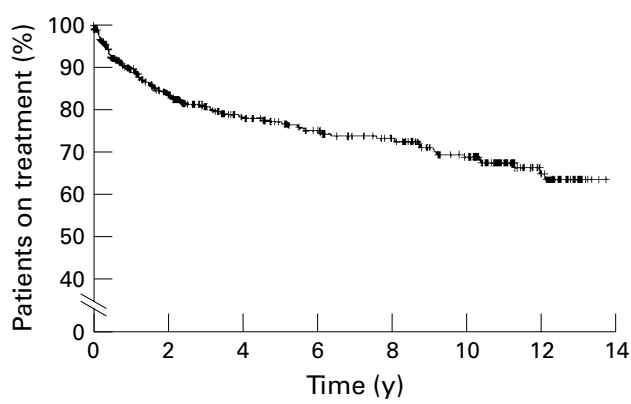

Figure 3 Time to first treatment change: all drugs (addition or substitution of drug to existing treatment).

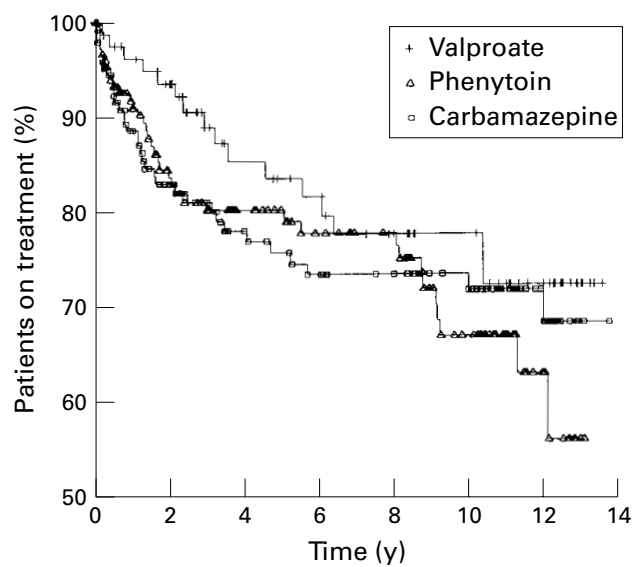

Figure 4 Time taken to first treatment change: all drugs (addition or substitution of drug to existing treatment).

STATISTICAL METHODS

Kaplan-Meier survival analyses were carried out using SPSS version 7.5.1, to estimate the time taken to change treatment for the first time, by either adding a drug or substituting a drug. The time taken to first change of individual drugs and the percentage of patients continuing on a particular drug were also calculated using the same method. Life table survival analyses were carried out to estimate the total number of patients continuing on medication at the end of the follow up period.

\section{Results}

Out of 564 patients with newly diagnosed epilepsy, only 17 were lost to follow up at the end of the study in 1997 (the median follow up was 11.8 years (25th and 75 th percentiles 10.6 and 12.7 years respectively) a total of 11400 person years). The causes of loss to follow up in these 17 patients were emigration (four), entry into the armed forces (five), withdrawal of consent (three), and unknown (five). Four hundred and thirty three $(77 \%)$ were started on drug treatment. Of the $131(23 \%)$ who did not start therapy, 41 had had acute symptomatic seizures; 42 died within the first 6 months due to underlying diseases. Of the 252 patients where the index seizure was also the first seizure, only $38(15 \%)$ were prescribed medication after the first seizure. Due to high rate of seizure recurrence in these patients, however, almost $70 \%$ were eventually treated. Initial therapy was prescribed by a hospital physician in 303 $(70 \%)$ cases and a GP initiated therapy in the remainder (although often on the advice of a specialist).

Four hundred and thirty three (77\%) patients were started on therapy. Phenytoin was started in 161/564 (29\%), carbamazepine in $154 / 564(27 \%)$, valproate in $84 / 564(15 \%)$, phenobarbital in $14 / 564(2 \%)$, and other drugs including benzodiazepines and ethosuximide in $20 / 564(4 \%)$. Of these, $426(98 \%)$ were started on single drug therapy and seven $(2 \%)$ patients were started on duotherapy, three with phenytoin and phenobarbital, and four patients with phenytoin and carbamazepine.

Of 292 patients with partial seizures with or without secondary generalisation, $236(81 \%)$ received treatment. Of these, 105 (44\%) patients were prescribed carbamazepine, 90 $(38 \%)$ phenytoin, $27(11 \%)$ valproate, and 14 $(6 \%)$ received other drugs. Of the 160 out of 221 patients with generalised seizures who received treatment, phenytoin was the most often preferred drug in $59(37 \%)$ patients followed by valproate in $48(30 \%)$, carbamazepine in $44(28 \%)$, and other drugs in nine $(6 \%)$. Carbamazepine was prescribed initially in nine patients who had absence and/or myoclonic seizures in addition to generalised tonic-clonic seizures. Of 36 patients with unclassified seizures, phenytoin was the most often preferred drug (36\%) followed by valproate $(25 \%)$, carbamazepine $(19.5 \%)$, and other drugs (19.5\%).

A life table survival analysis (fig 1) of patients shows that patients withdrawing from therapy usually do so early in the course of their history. The sequence of treatment events is illustrated in figure 2 . Ninety one of $564(16 \%)$ patients changed medication for the first time because of lack of efficacy. Of these 91, 29 (32\%) patients have now achieved 2 year terminal remission and $21(23 \%)$ have achieved 5 year terminal remission. Five per cent (30) of all patients went on to change medication for the second time for the same reason, and of this group nine patients $(30 \%)$ achieved 2 year terminal remission and only five $(17 \%)$ have achieved 5 year terminal remission. Of the 50/564 (9\%) patients who changed for the first time due to adverse events, only four did so again for the same reason. Thirty nine of 564 patients $(7 \%)$ have not switched to any other drug despite failure to attain even 2 year terminal remission from seizures.

A new antiepileptic drug such as gabapentin, lamotrigine, vigabatrin, or topiramate was used in only $25(11 \%)$ treatment changes in 17 patients with recurrent seizures, out of a total of 221 treatment changes in the whole cohort $(11 \%)$. The most commonly used new antiepileptic drugs were lamotrigine and vigabatrin (11 patients each), followed by gabapentin (two patients) and topiramate (one patient).

Kaplan-Meier survival analysis estimated the time course of treatment changes (addition or substitution of antiepileptic drugs) in the cohort (fig 3) Of the 433 treated patients, 15\% changed their initially prescribed medication for the first time within 2 years and a further $20 \%$ did so in the next 12 years. About $18 \%$ of patients on phenytoin and carbamazepine 


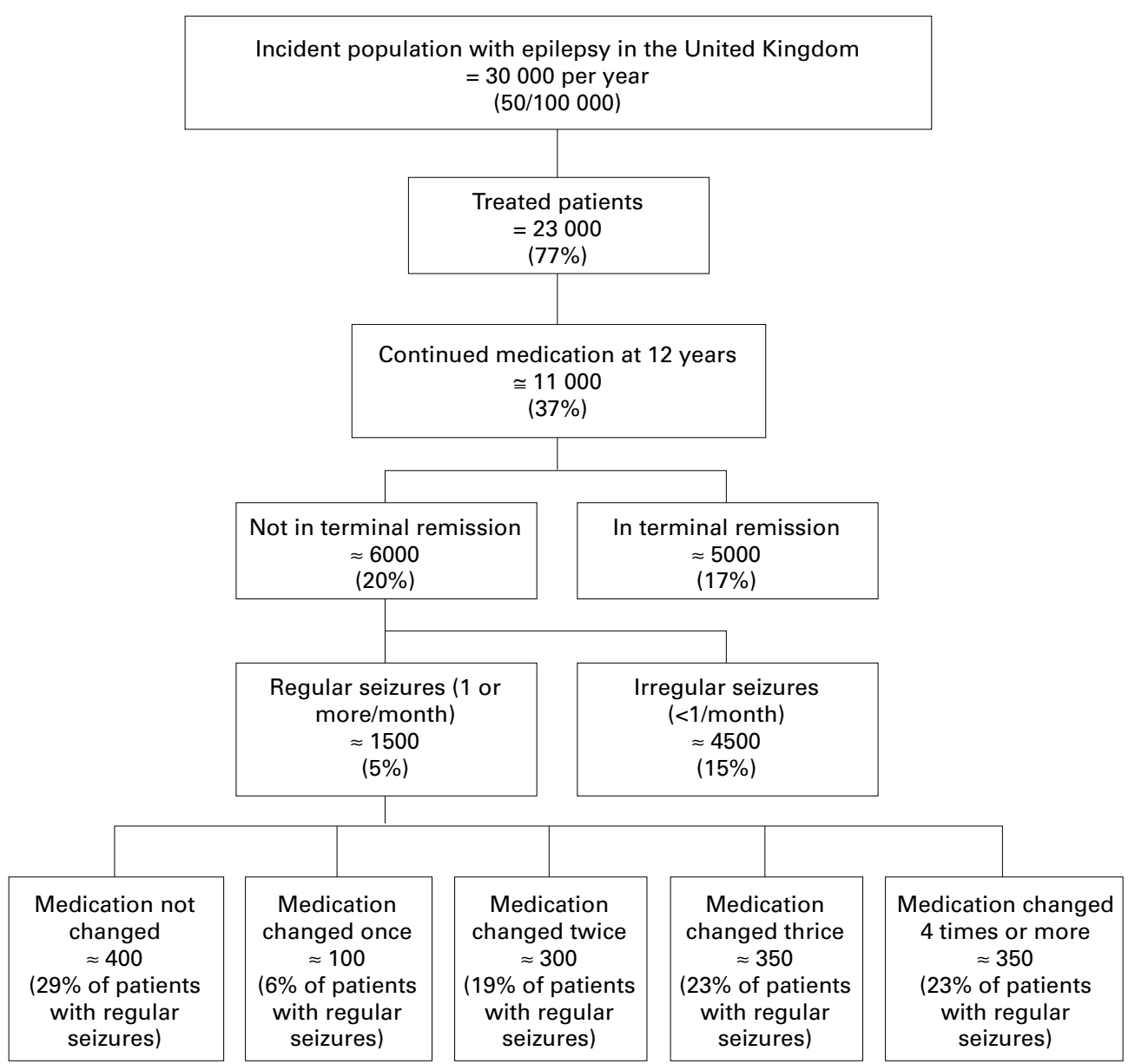

Figure 5 A model of long term treatment patterns in epilepsy in the United Kingdom.

changed medication in the first 2 years compared with $7 \%$ on valproate (fig 4 ).

Two hundred and nine of 564 patients $(37 \%)$ were on drug therapy for epilepsy at the time of last follow up; 168/564 patients (30\%) stayed continuously on medication and another $41 / 564$ patients $(7 \%)$ restarted drug therapy because of seizure recurrence, having withdrawn medication; 42/564 (7\%) were on combined therapy with two drugs and only $15 / 564(3 \%)$ patients were on three or more drugs simultaneously at the time of last follow up. Ninety eight of those on treatment are known to be in 5 year terminal remission.

One hundred and eleven of 564 patients $(20 \%)$ had not achieved 5 year terminal remission. Thirty nine of 564 patients $(7 \%)$ had not had any drug switches despite lack of remission and continued on their originally prescribed medication. Thirty one of 564 patients $(5 \%)$ continued to have frequent (one or more a week) seizures.

Nine of 31 patients (29\%) with frequent seizures had changed drug doses but had not switched to any other drug. Two of $31(6 \%)$ patients had changed their initially prescribed drug once, 6/31 (19\%) had done so twice and another $7 / 31(23 \%)$ had done so thrice. Only $7 / 31$ patients $(23 \%)$ had changed four times or more. Thus, only 22 out of 31 patients with frequent seizures at the time of follow up (4\% of the study population) changed medication at least once to achieve better seizure control.

\section{Discussion}

We report here an observational study of treatment patterns over 11-14 years of follow up in an unselected population of patients with newly diagnosed epilepsy. We found patterns but did not influence therapy (as the study team were not the physicians involved in the patient care). We are unaware of any similar such study or data. This study provides a useful overview of the dynamics of treatment and treatment changes, as they occur in routine practice throughout the United Kingdom. These data are valuable for targeting health service provision and identifying areas for raising clinical standards. The design of the NGPSE has allowed a robust, evidence based analysis ${ }^{5}$ of the recurrence, ${ }^{6}$ remission, ${ }^{7}$ prognosis, ${ }^{8}$ and mortality ${ }^{9}$ of newly diagnosed epilepsy in an unselected population. This analysis of treatment dynamics in the NGPSE discloses several interesting findings that provide a picture of the patterns of treatment of epilepsy in the general population during the period 1984-97, a pattern of treatment which is quite different from that found from hospital clinics of chronic, often intractable cases. We 
found that $77 \%$ of patients receiving a diagnosis of epilepsy are started on therapy. The hospital physician (clinic and accident and emergency) initiated therapy in over two thirds of patients. Furthermore, it is likely that in many of the patients in whom therapy was started by the GP, the advice of the hospital was taken. This seems to be a change in practice over the past decade, ${ }^{2}$ and is an improvement in line with current guidelines, which emphasise the importance of obtaining specialist advice before initiating antiepileptic drug therapy.

The study allowed us to track treatment over the 12 year period from the initiation of therapy. Overall, about one third of patients continued long term therapy (were still on treatment at the time of last follow up, a mean of 12 years). The yearly incidence of epilepsy is about 50/100 000 in the United Kingdom or 30000 patients a year. Thus, about 11000 newly diagnosed patients each year continue to be on medication for a mean of 12 years after the initiation of therapy (fig 5). Of these, about 5000 continue on drug therapy despite long term remission from seizures and about 6000 do so because of unsatisfactory seizure control. Most of the second group have infrequent seizures although about 1500 patients have seizures at least once a month. About 2000 patients will not have changed their medication in this period. Of these, surprisingly, 400 patients will not have done so despite frequent seizures. Of the patients having more than one seizure a week, one third are still taking their initial antiepileptic drug. Only about 400 patients may have tried four or more drugs, alone or in combination, in addition to the initial medication for seizure control. When the fact that these are annual, recurring figures in each year's incident population is considered, it is probable that there are many people in the prevalent "pool" of patients with chronic epilepsy who have not been adequately treated. The lack of change and the slowness to change are an indictment of current practice, and suggest that too often an insouciant attitude is taken to early epilepsy. How many of the $20 \%$ of patients with continuing epilepsy would have benefited from a more aggressive approach to treatment is not possible to say, but almost certainly some medical and social complications could have been avoided, and perhaps even mortality.

Having said this, the outcome of those who do make treatment switches is not necessarily good. One third of those who did switch because of poor control on the first drug, failed to gain control on the second. Furthermore, only one sixth of those who switched because of poor control on the second drug obtained lasting control on the third. Although it is possible that alternative drug changes would have helped some of these patients, most are likely to be resistant to all antiepileptic drugs. The first switch was usually within 2 years of initiating therapy. These are the types of patients who develop chronic epilepsy. As the first drug change is usually in the first 2 years after diagnosis, our findings imply that the patients at risk of chronicity can be identified early in the course of their epilepsy. In most, but not all, patients, the failure of initial monotherapy is predictive of chronicity, as has been noted before. ${ }^{10}$ The identification of reliably predictive clinical factors at this stage would be of great importance, but to date none have been found. More research is needed to identify these drug resistant patients earlier, as more aggressive therapy or much earlier surgical intervention would be appropriate in many.

About a quarter of patients with newly diagnosed epilepsy did not receive medication for their seizures, most commonly those with serious or progressive underlying disease. Only $15 \%$ of patients whose index seizure was also their first seizure received medication after the first seizure. This reflects a more conservative approach to single seizure management in the United Kingdom, in contrast to about $70 \%$ of such patients in the United States who are given drug treatment ${ }^{11}$; which is the most appropriate approach is controversial. However, because of high rates of subsequent seizure recurrence, the ultimate number who received antiepileptic medication was more than $70 \%$.

The vast majority of patients were prescribed initial monotherapy. This is in accord with current guidelines. Therapy with more than one drug has been recommended in the past, ${ }^{12}$ although the lack of evidence supporting this has been pointed out. ${ }^{13}$ Duotherapy no longer seems to be common practice as only seven patients were simultaneously started on two drugs. With continued drug treatment, polytherapy was more common although less than 3\% continued on three or more drugs. Less than $10 \%$ of all patients continued on two drugs, suggesting that this is a relatively infrequent practice in an unselected population with epilepsy, contrary to findings in more selected populations with uncontrolled seizures. ${ }^{14}$

The choice of drug for initial therapy in this study is a useful reflection on overall practice in the United Kingdom between 1984 and 1987. Phenytoin and carbamazepine were the most often preferred first line drugs and were used in over a third each of the treated cohort; valproate was used in a fifth and phenobarbital in only a minority. The comparatively infrequent use of phenobarbital is probably due to increased awareness of its frequent adverse events and the availability of better drugs and this is a change in practice from that a decade earlier. Carbamazepine was the most commonly used first line drug in partial seizures, consistent with current advice. ${ }^{15}$ Only a slightly smaller number however, were prescribed phenytoin, suggesting that this is also a popular first choice drug. In the case of generalised seizures, phenytoin was most often prescribed. Only a third of treated patients with generalised epilepsy were prescribed valproate first, contrary to its current status as first line therapy. ${ }^{16}{ }^{17}$ Furthermore, carbamazepine was used in nine patients who had idiopathic generalised epilepsy and absence and/or myoclonic seizures. In such situations, carbamazepine is a contraindication because of the risk of exacerbating seizures. ${ }^{18}$ Almost all the patients with childhood absence epilepsy 
were prescribed ethosuximide. Although this remains effective therapy for the condition, valproate is probably a more favoured first line option by many specialists nowadays. ${ }^{16}$

Most patients who change therapy due to adverse events or lack of efficacy seem to do so in the first 2 years (fig 3$)$. Valproate $(7 \%)$ seemed to be less commonly discontinued at 2 years than carbamazepine $(18 \%)$ or phenytoin $(19 \%)$ (fig 4). Less than $10 \%$ of the cohort patients changed medication for the first time because of adverse events, suggesting that this is not an overly frequent cause of failed drug retention (fig 1). This figure is similar to another study of drug therapy in newly diagnosed epilepsy although only hospital referred patients with two or more seizures were considered over a 3 year period. ${ }^{19}$ Only four patients subsequently changed therapy again because of adverse events to the second drug and repeated discontinuation of medication due to adverse events seems uncommon in the general population. Lack of efficacy is the commonest cause for change of therapy and $16 \%$ of the cohort did so for the first time for this reason. A third of these patients went on to change therapy again for the same reason, suggesting more refractory seizures in these patients.

Even at the stage of the first treatment change, several patients had medication added rather than substituted. Substitution however, was four times more frequent. Current practice is to advise that monotherapy with the conventional antiepileptic drugs should be tried sequentially before trying combination therapy.

Only $11 \%$ of treatment changes involved a new antiepileptic drug (lamotrigine, vigabatrin, gabapentin, and topiramate). This is unsurprising as most drug changes took place in the first 2 years of entering into the study, which was before these antiepileptic drugs were widely available. The situation is probably gradually changing now.

Thirty eight patients who continued on therapy did so despite 5 or more years of terminal remission. Driving considerations, fear of seizure recurrence, and lack of specialist advice on drug discontinuation may account for this. Of the 111 patients not in 5 year terminal remission, $31(28 \%)$ had one or more seizures a week at the time of last follow up and although a high frequency of drug changes would be expected in this group, a third had only tried a single drug. Only a quarter had changed their medication four or more times. This may suggest poor specialist input in this group as more than a third were not under regular hospital follow up despite frequent seizures.

In summary, this study allows us to determine to what extent the pattern of therapy in the population follows accepted clinical guidelines. Practice in some areas is acceptable; for instance, the widespread use of initial monotherapy and substitution not addition of the second drug; hospital referral for initial treatment advice; restriction of initial monotherapy to conventional cheaper drugs; and a general reluctance to treat the first seizure. Other aspects of practice are below acceptable standards-for instance, lack of change of medication in the face of continuing seizures; lack of specificity in the choice of drugs; and reluctance to use newer antiepileptic drugs in patients with resistant epilepsy. There also seems to be a lack of correlation between occurrence of seizures and the decision to continue or withdraw therapy. Some patients may have benefited from the newer antiepileptic drugs although it is probable that only a small proportion of patients in the general population require these drugs, and this is in marked contrast to those in hospital clinics. Our findings indicate that there is considerable scope for improving the care of patients with epilepsy, that treatment is not changed rapidly or often enough in those with continuing seizures. This lack of urgency will inevitably result in less good medical and social outcomes for people with epilepsy. The findings of our survey are therefore somewhat of a curate's egg. Although it is clear that some aspects of therapy are better than in previous surveys, others are not, and there is probably considerable room for improvement in the provision of epilepsy treatment in the United Kingdom.

We thank the Brain Research Trust, the National Society for Epilepsy, the National Hospital for Neurology and Neurosurgery, and Action Research for grants to support the NGPSE.

1 Sander JWAS, Shorvon SD. Epidemiology of the epilepsies. 7 Neurol Neurosurg Psychiatry 1996;61:443.

2 Hart YM, Shorvon SD. The nature of epilepsy in the general population. I. Characteristics of patients receiving medication for epilepsy. Epilepsy Res 1995;21:43-9.

3 Hart YM, Sander JWAS, Shorvon SD. National General Practice Study of Epilepsy and Epileptic seizures: Objectives and study methodology of the largest reported prospective cohort study of epilepsy. Neuroepidemiology 1989;8:221-227.

4 The Commission on classification and terminology of the International League Against Epilepsy. Proposal for revised clinical and electroencephalographic classification of epileptic seizures. Epilepsia 1981;22:489-501.

5 Evidence Medicine Working Group. Evidence based medicine: a new approach to teaching the practice of medicine. ҰAMA 1992;268:2420-5.

6 Hart YM, Sander JW, Johnson AL, et al. National general practice study of epilepsy: recurrence after a first seizure. Cocket 1990:336:1271-4.

7 Cockerell OC, Johnson AL, Sander JW, et al. Remission of epilepsy: results from the National general practice study of epilepsy. Lancet 1995;346:140-4

8 Cockerell OC, Johnson AL, Sander JW, et al. Prognosis of epilepsy: a review and further analysis of the first 9 years of the British national general practice study of epilepsy, a prospective population-based study. Epilepsia 1997;38:3146.

9 Cockerell OC, Johnson AL, Sander JW, et al. Mortality from epilepsy: results from a prospective population-based study. Lancet 1994;344:918-21.

10 Shorvon SD. The temporal aspects of prognosis in epilepsy. 7 Neurol Neurosurg Psychiatry 1984;47:1157-65.

11 Hauser WA, Andersen VE, Loewenson RB, et al. Seizure recurrence after a first unprovoked seizure. $N$ Engl $\mathcal{F} \mathrm{Med}$ 1982;307:522-8

12 Yahr MD, Sciarra D, Carter S. Evaluation of standard anticonvulsant therapy of 319 patients. $\mathscr{f} A M A$ 1952;150:6637 .

13 Shorvon SD, Chadwick D, Galbraith AW, et al. One drug for epilepsy. BMF 1978;1:474-6.

14 Mattson RH. Drug treatment of uncontrolled seizures. Epilepsy Res 1992;(suppl 5):29-35.

15 Sillanpaa M. Carbamazepine and oxcarbazepine. In: Shorvon SD, Dreifuss F, Fish DR, et al, eds. The treatment of epilepsy. Oxford: Blackwell, 1996:403-13.

6 Bruni J. Valproate. In: Shorvon SD, Dreifuss F, Fish DR, et al, eds. The treatment of epilepsy. Oxford: Blackwell, 1996:482-90.

17 Mattson RH, Cramer JA, Collins JF. A comparison of valproate with carbamazepine for the treatment of complex partial seizures and secondarily generalised tonic-clonic seizures in adults. The Department of Veteran Affairs Epilepsy Cooperative Study No 264 Group. $N$ Engl $f$ Med 1992;327:765-71.

18 Snead OC, Hosey LC. Exacerbation of seizures in children by carbamazepine. N Engl f Med 1985;313:916-21.

19 Heller A, Chesterman P, Elwes RD, et al. Phenobarbitone, phenytoin, carbamazepine, or sodium valproate for newly diagnosed adult epilepsy: a randomised comparitive monotherapy trial. $\mathcal{F}$ Neurol Neurosurg Psychiatry 1995;58:44-50. 